\title{
Investigation of Confounding Factors in Measuring Tissue Saturation with NIRS Spatially Resolved Spectroscopy
}

\author{
Z. Kovacsova, G. Bale, S. Mitra, I. de Roever, \\ J. Meek, N. Robertson, and I. Tachtsidis
}

\begin{abstract}
Performing absolute measurements of tissue saturation of the brain with near-infrared spectroscopy (NIRS) is a clinically desirable brain monitoring tool. Tissue oxygenation index (TOI) is an indicator of absolute tissue mixed arterial and venous oxygen saturation, and can be calculated using a NIRS technique called spatially resolved spectroscopy (SRS). SRS instruments measure the change of light attenuation with distance by using multiple light source-detector distances at two or more wavelengths. The aim of the study is to use broadband NIRS SRS data to investigate the effects on the calculation of TOI of different parameters: wavelength selection, scattering dependence, source-detector distance, and resolving for water. In total, 55 neonates with hypoxic-ischemic encephalopathy were monitored using a broadband multi-distance continuous wave NIRS system; 172 datasets were recorded. Using a "Standard" approach, TOI values between 0 and $100 \%$ ("good") were calculated in $157 / 172$ datasets with a mean
\end{abstract}

Z. Kovacsova $(\bowtie) \cdot$ G. Bale $\cdot$ I. de Roever I. Tachtsidis

Medical Physics \& Biomedical Engineering,

UCL, London, UK

e-mail: zuzana.kovacsova.15@ucl.ac.uk

S. Mitra $\cdot$ J. Meek $\cdot$ N. Robertson

Department of Neonatology, Institute for Women's

Health, UCL, London, UK
TOI of $50 \%$. By changing the wavelength selection, the number of "good" data sets increases to $165 / 172$ with a mean of $60 \%$. Alteration of the dependence of scattering on wavelength acts as a constant which shifts the absolute value of TOI significantly ( $p<0.05)$, demonstrating the importance of having a subject-appropriate estimation of scattering dependence. In general, changing the combination of source-detector distances does not significantly alter the TOI (the mean TOI ranges from $41 \%$ to $53 \%$ ) which suggests that the algorithm is robust to different sourcedetector combinations. The study shows the broadband NIRS SRS algorithm gives the opportunity to explore the calculation of TOI and could further improve the measurement of tissue saturation in a clinical setting.

\section{Introduction}

Near-infrared spectroscopy (NIRS) is a noninvasive clinical monitoring method which provides real-time information on the oxygenation and haemodynamics of tissue. It is based on the absorption of near-infrared light by haemoglobin; as the absorption properties of haemoglobin change with its oxygenation status, measuring the transmitted/reflected light from tissue informs on its oxygenation state of tissue. Using NIRS to 
monitor absolute tissue oxygenation in the brain is clinically desirable as it can inform on the balance between cerebral blood flow and oxygen supply. NIRS can measure brain tissue oxygen saturation $\mathrm{StO}_{2}$, also known as Tissue Oxygenation Index (TOI), which is the ratio of the concentration of oxyhaemoglobin $\mathrm{HbO}_{2}$ and total haemoglobin $\mathrm{HbT}=\mathrm{HbO}_{2}+\mathrm{HHb}$, where $\mathrm{HHb}$ is deoxyhaemoglobin. One of the methods to measure absolute tissue oxygenation is spatially resolved spectroscopy (SRS). SRS instruments are able to measure the change in light attenuation $A$ with distance $\rho$ by using two or more source-detector separations. A scaled absorption coefficient $k \mu_{a}$ is calculated using the slope of $A$ :

$$
\mathrm{k} \mu_{\mathrm{a}}=\frac{1}{3(1-h \lambda)}\left(\ln (10) \frac{\partial A(\lambda)}{\partial \rho}-\frac{2}{\rho}\right)^{2}
$$

where $h$ is the gradient of the transport scattering coefficient $\mu_{s}^{\prime}$ with wavelength $\lambda ; \mu_{s}^{\prime}=k(1-h \lambda)$. Scaled concentration of haemoglobin $k\left[\mathrm{HbO}_{2}\right]$ and $k[\mathrm{HHb}]$ are quantified; the scaling factor $k$ (which is unknown from the measurement) cancels itself when expressing oxygenation in terms of TOI, i.e. TOI $=k\left[\mathrm{HbO}_{2}\right] / k[\mathrm{HbT}][1]$.

\section{Method}

The data in this study were previously published by Bale et al. [2]. A broadband multi-distance continuous wave NIRS system, CYRIL, CYtochrome Research Instrument and appLication (source-detector distances: 30, 25, 20, $15 \mathrm{~mm}, 770-906 \mathrm{~nm}$ ) [3], was used to monitor the right frontal hemisphere of neonates with hypoxic-ischaemic encephalopathy during the first 4 days of life. The patients were diagnosed with severe or moderate brain injury and all were treated with therapeutic hypothermia for $48 \mathrm{~h}$. Data were recorded from 55 neonates and yielded 172 separate datasets (durations from $10 \mathrm{~min}$ to $18 \mathrm{~h}$ ). To minimize computational burden, datasets were cut to be $600 \mathrm{~s}$ long; the time window was 200-799 s. The first 199 s of the measurement were excluded to avoid set-up errors. The
"Standard" SRS method (described below) produced, in some cases, TOI values which were negative or above $100 \%$. We now attempt to correct these values by investigating the SRS algorithm and how the input parameters influence TOI. The different approaches to resolve TOI were:

- Approach 0, "Standard": This SRS approach resolved for only haemoglobin chromophores from $k \mu_{a}$; the wavelength dependence of the scattering coefficient was $h=0.00063 \mathrm{~mm}^{-1} \mathrm{~nm}^{-1}[1,4]$; the entire broadband spectrum of the NIRS data (770$906 \mathrm{~nm}$ ) was used; and all four detectors were used to quantify the attenuation slope.

- Approach 1, "Water": This approach additionally resolved for the water concentration from $k \mu_{a}$, i.e. water was treated as a third chromophore like $\mathrm{HbO}_{2}$ and $\mathrm{HHb}$. It does not obtain an absolute measure of the concentrations as $k$ is unknown.

- Approach 2, "Scattering": The dependence of scattering on wavelength as described by $h$ was modified. The "Standard" approach uses $h=0.00063 \mathrm{~mm}^{-1} \mathrm{~nm}^{-1}$ measured by Matcher et al. on adults [2] which is used in commercial systems [4]. In this approach, two other values are used. Highton et al. measured a value of $h=0.00085 \mathrm{~mm}^{-1} \mathrm{~nm}^{-1}$ in adults with traumatic brain injury [5]. Kurata et al. measured optical properties of the neonatal brain [6], a value of $h=0.00048 \mathrm{~mm}^{-1} \mathrm{~nm}^{-1}$ was calculated from the data for term infants using the method described by Matcher et al. [4].

- Approach 3, "Distance": The number and combination of source-detector separations was adjusted. A minimum of two sourcedetector distances are necessary for SRS; increasing their number leads to a more accurate approximation of the gradient of attenuation with distance. In this approach, the effect of using less detectors and applying various combinations of the detectors was studied; there were ten possible combinations.

- Approach 4, "Wavelengths": The "Standard" approach uses the full broadband spectrum; however, it is possible to measure TOI with a 
minimum of two wavelengths. The selected wavelengths were $775,810,850,905 \mathrm{~nm}$ or $775,810,850 \mathrm{~nm}$ which were chosen because they are used in commercial systems; NIRO 300, NIRO 200 (Hamamatsu Photonics, Japan).

Each approach was investigated independently from the others; the other parameters remained as in the "Standard" SRS approach. Approaches "Scattering", "Distance" and "Wavelength" have several sub-approaches. In total, there were 16 approaches resulting in 16 TOI values calculated from each dataset.

The resulting TOIs were averaged over the time window and compared to the "Standard" average TOI. A TOI value was considered to be "good" if it was between $0 \%$ and $100 \% .^{1}$ The number of "good" TOIs was counted for all approaches and sub-approaches. Minimum and maximum TOIs were found for all "good" TOIs out of the 172 datasets. Datasets in which all 16 TOI values were "good" were further analysed using SPSS Statistics (IBM 2016). A Sign test was applied to evaluate differences between "Standard" and every other approach, significance threshold $\mathrm{p}<0.05$. Spearman's rho $\left(\rho_{s}\right)$ was calculated between the "Standard" TOIs and every other approach to assess their correlation. The combination of analysing the statistical difference between approaches and their correlation can give us information about their similarity, for example:

- if $\rho_{s}$ is low and there is a significant difference between the approaches, then these are not similar.

- when $\rho_{s}$ is high and there is a significant difference between the approaches, then these are highly associated but have different absolute TOI values.

- if $\rho_{s}$ is high and there is not a significant difference between the approaches then these approaches are similar.

\footnotetext{
${ }^{1}$ This was used to exclude physiologically impossible values. At this stage of the study, it was not evaluated whether the TOI was a correct estimation of the brain oxygenation of the patient.
}

\section{Results}

Table 1 shows the calculated TOI values with the percentage of "good" TOI values for each SRS approach. The number of datasets which give "good" TOIs changes per approach; the most successful one is "Wavelengths $(775,810,850$ nm)" with 165/172 "good" TOIs and the least successful is "Distance $(15,20 \mathrm{~mm})$ " with $95 / 172$.

For 48 out of 172 datasets all 16 approaches gave "good" TOI values. Figure 1 shows a boxplot of the median TOI values with Spearman's Rho for each approach.

The Sign test showed that eight approaches gave significantly different results from the "Standard" ( $p<0.05)$. Those were: "Water", "Scattering" (both $h$ values), "Distance" (20,15 mm; 30,15 mm; 25,20 mm) and "Wavelengths" (both sets). Spearman's Rho was below 0.7 in six different instances, which suggests low association with the "Standard" approach: "Water" $\left(\rho_{s}=0.56\right)$, "Distance" $\left(\rho_{s}\right.$ $(20,15 \mathrm{~mm})=0.65, \rho_{s}(30,20 \mathrm{~mm})=0.64, \rho_{s}$ $\left.(30,25 \mathrm{~mm})=0.36, \rho_{s}(30,25,20 \mathrm{~mm})=0.64\right)$ and "Wavelength" $\left(\rho_{s}(775,810,850 \mathrm{~nm})=0.65\right)$.

\section{Discussion}

The aim of this study was to investigate the SRS algorithm using a multi-distance broadband NIRS device. The parameters investigated were: resolving for water, the scattering dependence on wavelength, source-detector distance combinations and wavelength selection. We have shown that TOI values vary significantly depending on the SRS approach.

To investigate the effect of changing the SRS approach, we quantified how many times a "good" TOI value (between 0 and 100\%) was estimated per dataset (Table 1). Changing the approach sometimes changes the TOI value from negative/above $100 \%$ to what we call "good" TOI (between $0 \%$ and 100\%). Even within "good" TOIs, the range of achievable values is broad. This is further explored by focusing on 48 datasets in which all 16 approaches result in "good" TOI - the median values significantly differ from 
Table 1 Number of "good" TOI values from the 172 datasets calculated using the different approaches

\begin{tabular}{|c|c|c|c|c|c|c|c|}
\hline Approach & Sub-approach & $\begin{array}{l}\text { Good data sets } \\
(/ 172)\end{array}$ & $\begin{array}{l}\text { Success } \\
{[\%]}\end{array}$ & $\begin{array}{l}\text { Mean TOI } \\
{[\%]}\end{array}$ & $\begin{array}{l}\text { Min TOI } \\
{[\%]}\end{array}$ & $\begin{array}{l}\text { Max TOI } \\
{[\%]}\end{array}$ & $\begin{array}{l}\text { SD } \\
{[\%]}\end{array}$ \\
\hline 0: Standard & & 157 & 91 & 50 & 6 & 96 & 16 \\
\hline 1: Water & & 161 & 94 & 62 & 28 & 96 & 10 \\
\hline \multirow{2}{*}{$\begin{array}{l}\text { 2: Scattering } \\
\left(\mathrm{mm}^{-1} \mathrm{~nm}^{-1}\right)\end{array}$} & 0.00085 & 143 & 83 & 66 & 22 & 96 & 15 \\
\hline & 0.00048 & 159 & 92 & 46 & 7 & 92 & 17 \\
\hline \multirow[t]{10}{*}{ 3: Distance (mm) } & 20,15 & 95 & 55 & 41 & 6 & 96 & 24 \\
\hline & 25,15 & 152 & 88 & 53 & 16 & 99 & 18 \\
\hline & 30,15 & 154 & 90 & 51 & 12 & 91 & 17 \\
\hline & 25,20 & 158 & 92 & 50 & 6 & 95 & 11 \\
\hline & 30,20 & 162 & 94 & 49 & 3 & 92 & 14 \\
\hline & 30,25 & 102 & 59 & 45 & 4 & 86 & 17 \\
\hline & $25,20,15$ & 151 & 88 & 53 & 16 & 96 & 18 \\
\hline & $30,20,15$ & 154 & 90 & 50 & 8 & 89 & 17 \\
\hline & $30,25,15$ & 149 & 87 & 51 & 6 & 97 & 18 \\
\hline & $30,25,20$ & 161 & 94 & 49 & 3 & 92 & 14 \\
\hline \multirow[t]{2}{*}{ 4: Wavelength $(\mathrm{nm})$} & $775,810,850,905$ & 163 & 95 & 53 & 14 & 90 & 14 \\
\hline & $775,810,850$ & 165 & 96 & 60 & 28 & 95 & 11 \\
\hline
\end{tabular}

Mean TOI, Min TOI, Max TOI and the standard deviation (SD) are calculated only from the "good" values

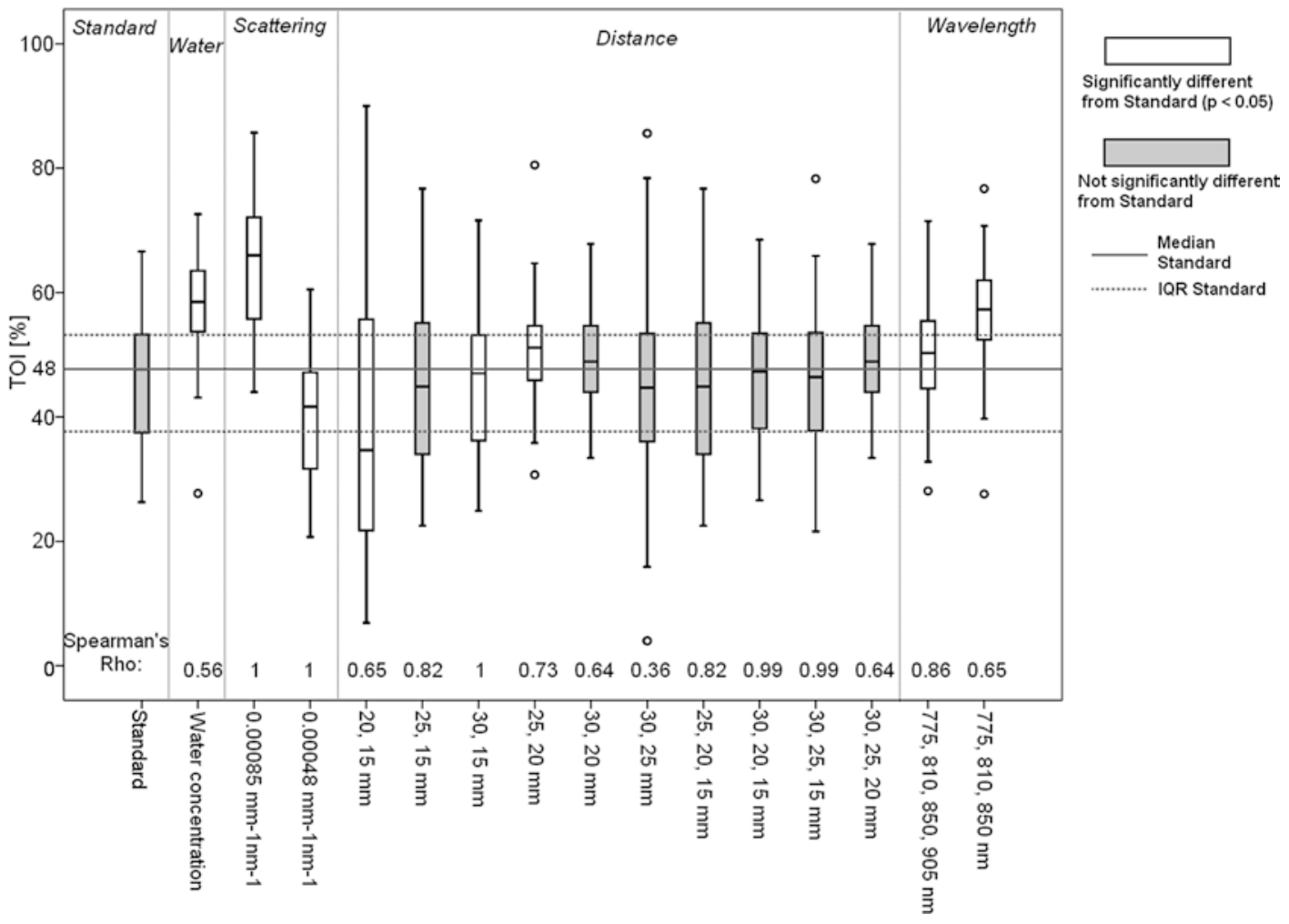

Fig. 1 Boxplot of median TOIs for the 48 datasets in which all approaches give "good" TOI values. The full line is the median of the "Standard" approach and the dotted lines are its inter-quartile range. Circles are outliers.
White boxes indicate significant difference $(\mathrm{p}<0.05)$ from the "Standard" approach. Spearman's Rho indicates the correlation between the "Standard" approach and other approaches 
"Standard" in the majority of approaches (Fig. 1). It is only the "Distance" approach, which gives similar results to "Standard" ( $p>0.05$ in $7 / 10$ distance combinations), which suggests that the algorithm is robust to different source-detector combinations.

The combination of the Spearman's Rho and the statistical significance assessments demonstrated low similarity between the "Standard" approach and: "Water", "Distance" (20, $15 \mathrm{~mm})$ and "Wavelength" (775,810,850 nm) approaches. However the approaches "Distance" (30,25,15 and $30,20,15 \mathrm{~mm}$ ) had the most similar results to the "Standard" approach.

The approach "Wavelength" shows that the selection of wavelength has a significant impact on the resulting TOI; this is important as different commercial brain oximeters (that apply the SRS method) use different wavelengths. For example OxyMon (Artinis Medical Systems, the Netherlands) uses 765 and $855 \mathrm{~nm}$, but NIRO200NX (Hamamatsu, Japan) uses 735, 810 and $850 \mathrm{~nm}$.

Significant shifts in TOI were caused by changing $h$; each value of $h$ was acquired in a different patient group: $0.00063 \mathrm{~mm}^{-1} \mathrm{~nm}^{-1}$ in 7 healthy adults, $0.00085 \mathrm{~mm}^{-1} \mathrm{~nm}^{-1}$ in 21 brain injured adults and $0.00048 \mathrm{~mm}^{-1} \mathrm{~nm}^{-1}$ in 8 fullterm neonates. This demonstrates the importance of having a subject-appropriate estimation of wavelength dependence of scattering. It has been shown that scattering properties change with age and pathology [6-8]; more investigations of the scattering dependence on wavelength and its behaviour during pathology are needed.

A broadband NIRS multi-distance device can provide additional information to improve the robustness of the SRS estimation of TOI, as it gives insight into the individual parameters in the algorithm and helps quantify their importance and impact. The limitation of this study is that the brain oxygenation of the studied patients is unknown and thus there is no physiological comparison.
The next steps include studying the effect on TOI by combining several approaches at once; and doing this using a dynamic blood phantom that allow us to control oxygenation (similar to a phantom described in [9]). We will do this while simultaneously measuring chromophore concentrations and oxygenation with a time-resolved system (TR). TR systems measure absolute concentrations of chromophores by resolving absorption and scattering [10]. This will enable us to further study and evaluate the effect of each approach and possibly identifying an optimal combination of parameters.

Acknowledgments This study was funded by BitMap, EU grant 675332 and by The Wellcome Trust, grant 104580/Z/14/Z.

\section{References}

1. Suzuki S, Takasaki S, Ozaki T et al (1999) A tissue oxygenation monitor using NIR spatially resolved spectroscopy. SPIE Proc 3597:582-592

2. Bale G, Mitra S, de Roever I et al (2016) Interrelationship between broadband NIRS measurements of cerebral cytochrome $\mathrm{C}$ oxidase and systemic changes indicates injury severity in neonatal encephalopathye. Adv Exp Med Biol 923:181-186

3. Bale G, Mitra S, Meek J et al (2014) A new broadband near-infrared spectroscopy system for in-vivo measurements of cerebral cytochrome-c-oxidase changes in neonatal brain injury. Biomed Opt Express 5(10):3450-3466

4. Matcher S, Kirkpatrick P, Nahid K et al (1995) Absolute quantification methods in tissue near infrared spectroscopy. Proc SPIE 2389:486-495

5. Phan P, Highton D, Lai J et al (2016) Multi-channel multi-distance broadband near-infrared spectroscopy system to measure the spatial response of cellular oxygen metabolism and tissue oxygenation. Biomed Opt Express 7(11):4424-4440

6. Kurata T, Iwata S, Tsuda K et al (2016) Physiological and pathological clinical conditions and light scattering in brain. Sci Rep 6(1):31354

7. Highton D, Tachtsidis I, Tucker A et al (2016) Near infrared light scattering changes following acute brain injury. Adv Exp Med Biol 876:139-144

8. Ijichi S, Kusaka T, Isobe K et al (2005) Developmental changes of optical properties in neonates determined 
by near-infrared time-resolved spectroscopy. Pediatr Res 58(3):568-573

9. Kleiser S, Nasseri N, Andresen B et al (2016) Comparison of tissue oximeters on a liquid phantom with adjustable optical properties. Biomed Opt Express 7(8):2973-2992
10. Torricelli A, Contini D, Pifferi A et al (2013) Time domain functional NIRS imaging for human brain mapping. NeuroImage 85:28-50

Open Access This chapter is licensed under the terms of the Creative Commons Attribution 4.0 International License (http://creativecommons.org/licenses/by/4.0/), which permits use, sharing, adaptation, distribution and reproduction in any medium or format, as long as you give appropriate credit to the original author(s) and the source, provide a link to the Creative Commons license and indicate if changes were made.

The images or other third party material in this chapter are included in the chapter's Creative Commons license, unless indicated otherwise in a credit line to the material. If material is not included in the chapter's Creative Commons license and your intended use is not permitted by statutory regulation or exceeds the permitted use, you will need to obtain permission directly from the copyright holder. 